International Journal of Pure and Applied Mathematics

Volume 92 No. 4 2014, 449-457

ISSN: 1311-8080 (printed version); ISSN: 1314-3395 (on-line version)

url: http://www.ijpam.eu

doi: http://dx.doi.org/10.12732/ijpam.v92i4.1

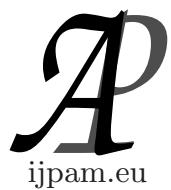

\title{
A DUALITY-BASED DERIVATION OF THE MAXIMUM FLOW FORMULATION OF THE OPEN-PIT DESIGN PROBLEM
}

\author{
Henry Amankwah ${ }^{1}$, Torbjörn Larsson ${ }^{2} \S$, Björn Textorius ${ }^{3}$ \\ ${ }^{1}$ Department of Mathematics \& Statistics \\ University of Cape Coast \\ GHANA \\ ${ }^{2,3}$ Department of Mathematics \\ Linköping University \\ SWEDEN
}

\begin{abstract}
The open-pit design problem is to decide which ore in a deposit to mine in order to maximize profit, subject to constraints on mining precedence. After discretizing the volume of the deposit, the open-pit design problem can be formulated as a maximum flow problem in a capacitated network, as shown by J.-C. Picard in 1976. His derivation is based on a restatement of the problem as a quadratic binary program. We give an alternative derivation of the maximum flow formulation, using only linear programming duality.
\end{abstract}

AMS Subject Classification: 90C10, 90C35, 90C90

Key Words: open-pit mining, maximal closure, maximum flow

\section{Introduction}

Open-pit mining is an operation whereby an ore deposit in the ground is excavated from the surface of the land. In the process of mining, a deeper

Received: November 21, 2012

(C) 2014 Academic Publications, Ltd.

$\S_{\text {Correspondence author }}$ url: www.acadpubl.eu 
and deeper pit is formed until the operation ends. To estimate the potential profitability of the mining operation, and for planning purposes, it is common practice to try to calculate the optimal final shape of the pit before the operation starts.

To design an optimal pit, the possible mining volume is partitioned into fixed-size blocks, usually in layers. By using geological information from drill cores, the value of each block is estimated. The cost of mining each block is also estimated, and a profit value can thus be assigned to each block, as illustrated in Figure 1. Since open-pit mining is performed from the surface and the pit walls may not collapse, the pit shape must comply with certain precedence restrictions. For example, in Figure 1, if the safe slope angle is $45^{\circ}$ and block 5 is to be removed, then we have to remove blocks 1,2 , and 3 , as well. The open-pit mine design problem is thus, deciding the blocks of a deposit to mine in order to maximize the total profit, while obeying mining precedence constraints. A real-life 3-D block model can easily contain millions of blocks and for each of them there are typically nine precedence constraints. This problem size makes the design problem computationally demanding.

\begin{tabular}{|c|c|c|c|}
\hline-1 & 3 & 3 & -2 \\
1 & 2 & 3 & 4 \\
\hline & 2 & 8 & \\
5 & 6 & \multicolumn{1}{|l}{} \\
\end{tabular}

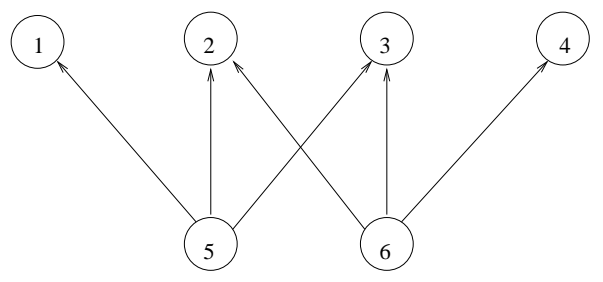

Figure 1: A 2-D block model of a mine with block profit values and its corresponding mine graph.

Lerchs and Grossmann [5] showed that the problem of determining an optimal pit can be stated as the problem of finding a maximal closure in a mine graph, associated with the block model of the mine, as illustrated in Figure 1. They also gave an algorithm for this problem, which has since been commonly used by the industry. Picard [6] showed that the maximal closure problem, and in particular the open-pit design problem, can be stated and solved as a maximum flow problem in a capacitated network that is constructed by augmenting the mine graph. Specifically, an optimal shape of the pit is given by a minimum cut in this maximum flow network. Later, Hochbaum and Chen [4] and Hochbaum [3] developed efficient maximum flow algorithms for the open-pit design problem. Some other contributions to the field of maximal closure and maximum flow are [7], [2], [10], and [8]. 
The purpose of this paper is to give an alternative derivation of the maximum flow formulation of the open-pit design problem. The derivation of Picard [6] is based on the restatement of the open-pit design problem as a quadratic binary program, while ours uses only standard linear programming duality, and is therefore more straightforward. In the next section we give the mathematical model for maximizing the total profit of a mine and in Section 3 the new derivation of the maximum flow problem is given.

\section{The Mathematical Model}

The following notations will be used.

$$
\begin{aligned}
V= & \text { set of all blocks that can be mined. } \\
A= & \text { set of pairs }(i, j) \text { of blocks such that block } j \text { is a } \\
& \text { neighbouring block to } i \text { that must be removed before } \\
& \text { block } i \text { can be mined. } \\
p_{i}= & \text { profit derived from mining block } i, i \in V .
\end{aligned}
$$

Defining the decision variables for all $i \in V$ as

$$
x_{i}=\left\{\begin{array}{l}
1, \text { if block } i \text { is mined, } \\
0, \text { otherwise, }
\end{array}\right.
$$

the model for maximizing the total profit is given by

$$
p^{*}=\operatorname{maximize} \sum_{i \in V} p_{i} x_{i}
$$

subject to

$$
\begin{aligned}
& x_{i} \leq x_{j}, \quad(i, j) \in A \\
& x_{i} \in\{0,1\}, \quad i \in V .
\end{aligned}
$$

\section{The New Derivation}

Consider the linear programming relaxation of Problem (1), obtained by replacing the condition $x_{i} \in\{0,1\}$ by $0 \leq x_{i} \leq 1$. The coefficients of the constraints of this linear program form a totally unimodular matrix, and it therefore possesses the integrality property, in that all the extreme points of the feasible set 
are integral [9]. The linear programming relaxation will therefore have the same optimal value. Further, if $p_{i}>0$ holds for some $i \in V$, then the lower bound constraint on that variable can never be binding, and it can therefore be relaxed without changing the optimal value. Similarly, if $p_{i} \leq 0$ holds, then the upper bound can be relaxed without any effect. We can thus rewrite Problem (1), in standard form, as

$$
p^{*}=\operatorname{maximize} \sum_{i \in V} p_{i} x_{i}
$$

subject to

$$
\begin{aligned}
& x_{i}-x_{j} \leq 0, \quad(i, j) \in A \\
& x_{i} \leq 1, \quad i \in V^{+} \\
& -x_{i} \leq 0, \quad i \in V^{-},
\end{aligned}
$$

where $V^{+}=\left\{i \in V \mid p_{i}>0\right\}$ and $V^{-}=\left\{i \in V \mid p_{i} \leq 0\right\}$.

Let $y_{i j}$, for $(i, j) \in A, w_{i}$, for $i \in V^{+}$, and $z_{i}$, for $i \in V^{-}$, be the linear programming dual variables (e.g. [1]) for the respective constraints, and introduce the sets $J_{i}^{+}=\{j \in V \mid(i, j) \in A\}$ and $J_{i}^{-}=\{j \in V \mid(j, i) \in A\}, i \in V$. The linear programming dual problem then becomes

$$
p^{*}=\operatorname{minimize} \sum_{i \in V^{+}} w_{i}
$$

subject to

$$
\begin{aligned}
& \sum_{j \in J_{i}^{+}} y_{i j}-\sum_{j \in J_{i}^{-}} y_{j i}+w_{i}=p_{i}, \quad i \in V^{+} \\
& \sum_{j \in J_{i}^{+}} y_{i j}-\sum_{j \in J_{i}^{-}} y_{j i}-z_{i}=p_{i}, \quad i \in V^{-} \\
& y_{i j} \geq 0, w_{i} \geq 0, z_{i} \geq 0, \quad(i, j) \in A, i \in V .
\end{aligned}
$$

Now, consider temporarily a fixed $i \in V^{+}$and the restriction of the Problem (2) obtained by adding the constraints $x_{j}=0$ for all $(j, i) \in A$ to the problem. From linear programming duality theory we then know that the restriction of (2) corresponds to a relaxation of Problem (3). With the values $x_{j}=0$ for all $(j, i) \in A$ in Problem (2) the constraints $x_{j}-x_{i} \leq 0,(j, i) \in A$, will clearly always be fulfilled and can therefore be dropped from the problem. This corresponds to setting $y_{j i}=0$ for all $(j, i) \in A$ in Problem $(3)$, and the constraint in (3) corresponding to $i \in V^{+}$reduces to

$$
\sum_{j \in J_{i}^{+}} y_{i j}+w_{i}=p_{i}
$$


This implies that $w_{i} \leq p_{i}$ holds in a relaxation of Problem (3) and thus it also holds in (3). By a similar argument we obtain that $z_{i} \leq-p_{i}$ holds for all $i \in V^{-}$. Hence, we can rewrite Problem (3) as

$$
p^{*}=\operatorname{minimize} \sum_{i \in V^{+}} w_{i}
$$

subject to

$$
\begin{aligned}
& \sum_{j \in J_{i}^{+}} y_{i j}-\sum_{j \in J_{i}^{-}} y_{j i}+w_{i}=p_{i}, \quad i \in V^{+} \\
& \sum_{j \in J_{i}^{+}} y_{i j}-\sum_{j \in J_{i}^{-}} y_{j i}-z_{i}=p_{i}, \quad i \in V^{-} \\
& w_{i} \leq p_{i}, \quad i \in V^{+} \\
& z_{i} \leq-p_{i}, \quad i \in V^{-} \\
& y_{i j} \geq 0, w_{i} \geq 0, z_{i} \geq 0, \quad(i, j) \in A, i \in V .
\end{aligned}
$$

Now, let

$$
v_{\sigma i}=p_{i}-w_{i}, \quad i \in V^{+} .
$$

Then $0 \leq v_{\sigma i} \leq p_{i}$ holds, since $0 \leq w_{i} \leq p_{i}$. Also, letting

$$
u_{i \tau}=-p_{i}-z_{i}, \quad i \in V^{-},
$$

we obtain that $0 \leq u_{i \tau} \leq-p_{i}$ holds, since $0 \leq z_{i} \leq-p_{i}$. Further, by (5) we have that

$$
\sum_{i \in V^{+}} w_{i}=-\sum_{i \in V^{+}} v_{\sigma i}+\sum_{i \in V^{+}} p_{i} .
$$

We next introduce the variable $f$, defined by

$$
f=\sum_{i \in V^{+}} v_{\sigma i} .
$$

From (7) and (8) it follows that the objective (4) can be replaced by a maximization of the variable $f$.

Adding the two equality constraints in the Problem (3) gives

$$
\sum_{i \in V^{+}} w_{i}-\sum_{i \in V^{-}} z_{i}=\sum_{i \in V} p_{i},
$$


since each variable $y_{i j}$ appears twice, with +1 and -1 coefficients. By (6), (9), and (7), we arrive at

$$
-\sum_{i \in V^{-}} u_{i \tau}=\sum_{i \in V^{-}} z_{i}+\sum_{i \in V^{-}} p_{i}=\sum_{i \in V^{+}} w_{i}-\sum_{i \in V^{+}} p_{i}=-\sum_{i \in V^{+}} v_{\sigma i}=-f .
$$

An equivalent formulation of Problem (3) is thus given by

$$
f^{*}=\text { maximize } \quad f
$$

subject to

$$
\begin{aligned}
& \sum_{i \in V^{+}} v_{\sigma i}=f \\
& \sum_{j \in J_{i}^{+}} y_{i j}-\sum_{j \in J_{i}^{-}} y_{j i}-v_{\sigma i}=0, \quad i \in V^{+} \\
& \sum_{j \in J_{i}^{+}} y_{i j}-\sum_{j \in J_{i}^{-}} y_{j i}+u_{i \tau}=0, \quad i \in V^{-} \\
& -\sum_{i \in V^{-}} u_{i \tau}=-f \\
& 0 \leq v_{\sigma i} \leq p_{i}, \quad i \in V^{+} \\
& 0 \leq u_{i \tau} \leq-p_{i}, \quad i \in V^{-} \\
& y_{i j} \geq 0, \quad(i, j) \in A,
\end{aligned}
$$

which can be interpreted as a maximum flow problem, in a network depicted in Figure 2, with $\sigma$ as the source node, $\tau$ as the sink node, $v_{\sigma i}$ as the flow on an arc from the source to node $i \in V^{+}, u_{i \tau}$ as the flow on an arc from node $i \in V^{-}$ to the sink, and $f$ as the total flow. It follows from $(3),(7)$, and (8) that

$$
p^{*}=\sum_{i \in V^{+}} p_{i}-f^{*} .
$$

Problem (10) and the equality (11) are consistent with the results of Picard [6].

To show, using our derivation, that an optimal solution to the pit design problem is given by a minimum cut for the maximum flow problem, we study the linear programming dual of Problem (10). Let $q_{\sigma}, q_{\tau}$, and $l_{i}, i \in V$, be the dual variables for the constraints for the source, the sink, and the block nodes, respectively. Let further $h_{i}, i \in V$, be the dual variables for the upper bound constraints. Then the dual of (10) will have the form 


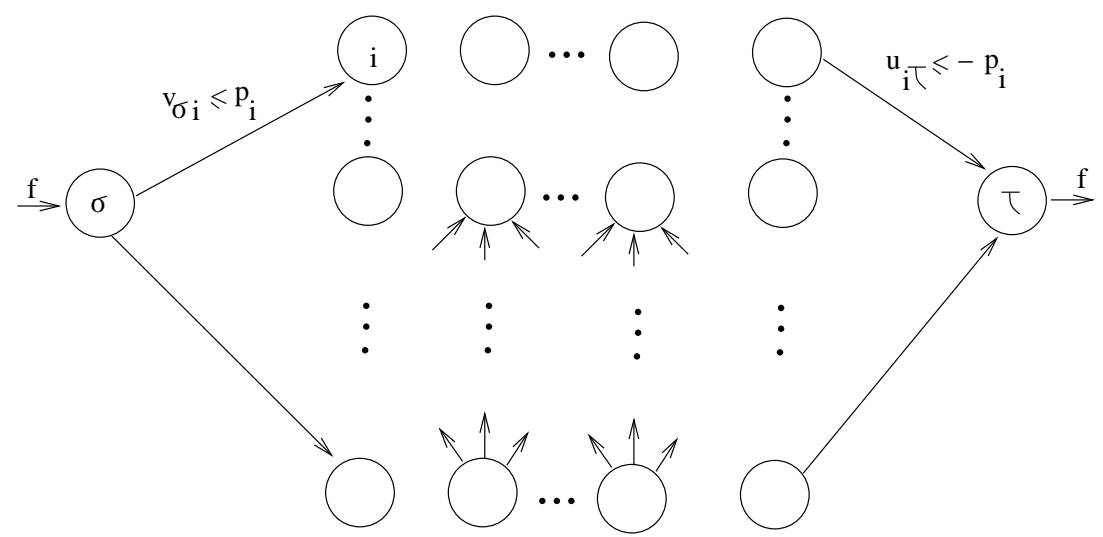

Figure 2: Maximum flow network.

$$
f^{*}=\operatorname{minimize} \sum_{i \in V^{+}} p_{i} h_{i}+\sum_{i \in V^{-}}\left(-p_{i}\right) h_{i}
$$

subject to

$$
\begin{aligned}
& q_{\tau}-q_{\sigma}=1 \\
& q_{\sigma}-l_{i}+h_{i} \geq 0, \quad i \in V^{+} \\
& l_{i}-q_{\tau}+h_{i} \geq 0, \quad i \in V^{-} \\
& l_{i}-l_{j} \geq 0, \quad(i, j) \in A \\
& h_{i} \geq 0, \quad i \in V .
\end{aligned}
$$

Let $(S, \bar{S})$, with $\sigma \in S$ and $\tau \in \bar{S}$, be a minimum cut. An optimal solution is then given by (e.g. [1]) $q_{\sigma}=0, q_{\tau}=1$,

$$
l_{i}^{*}=\left\{\begin{array}{ll}
0, & i \in S \\
1, & i \in \bar{S}
\end{array}, i \in V,\right.
$$

and

$$
h_{i}^{*}=\left\{\begin{array}{ll}
1, & i \in\left(V^{+} \cap \bar{S}\right) \cup\left(V^{-} \cap S\right) \\
0, & \text { otherwise }
\end{array}, i \in V .\right.
$$

Then, for $i \in V^{+}$,

$$
\left.\begin{array}{l}
i \in S \Longrightarrow h_{i}^{*}=l_{i}^{*}=0 \\
i \in \bar{S} \Longrightarrow h_{i}^{*}=l_{i}^{*}=1
\end{array}\right\} \Longrightarrow h_{i}^{*}=l_{i}^{*},
$$


and for $i \in V^{-}$,

$$
\left.\begin{array}{l}
i \in \bar{S} \Longrightarrow h_{i}^{*}=1-l_{i}^{*}=0 \\
i \in S \Longrightarrow h_{i}^{*}=1-l_{i}^{*}=1
\end{array}\right\} \Longrightarrow h_{i}^{*}=1-l_{i}^{*} .
$$

From (14) and (15) it follows that

$$
\begin{aligned}
f^{*} & =\sum_{i \in V^{+}} p_{i} h_{i}^{*}+\sum_{i \in V^{-}}\left(-p_{i}\right) h_{i}^{*}=\sum_{i \in V^{+}} p_{i} l_{i}^{*}+\sum_{i \in V^{-}}\left(-p_{i}\right)\left(1-l_{i}^{*}\right) \\
& =\sum_{i \in V} p_{i} l_{i}^{*}-\sum_{i \in V^{-}} p_{i} .
\end{aligned}
$$

Now, let $x_{i}^{*}=1-l_{i}^{*}$. Then (13) and (12) imply that $x_{i}^{*}, i \in V$, is feasible in Problem (1). By using (16) and (11) we obtain that

$$
\begin{aligned}
\sum_{i \in V} p_{i} x_{i}^{*} & =\sum_{i \in V} p_{i}\left(1-l_{i}^{*}\right)=\sum_{i \in V} p_{i}-\sum_{i \in V} p_{i} l_{i}^{*} \\
& =\sum_{i \in V^{+}} p_{i}-\sum_{i \in V} p_{i} l_{i}^{*}+\sum_{i \in V^{-}} p_{i}=\sum_{i \in V^{+}} p_{i}-f^{*}=p^{*}
\end{aligned}
$$

Since $x_{i}^{*}, i \in V$, is feasible, this implies that it is also optimal. Hence, an optimal solution to the pit design problem (1) is obtained from a minimum cut for the maximum flow problem (10).

\section{References}

[1] M.S. Bazaraa, J.J. Jarvis, Linear Programming and Network Flows, Wiley, New York, NY (1977).

[2] B. Faaland, K. Kim, T. Schmitt, A new algorithm for computing the maximal closure of a graph, Management Science, 36 (1990), 315-331.

[3] D.S. Hochbaum, A new-old algorithm for minimum-cut and maximum-flow in closure graphs, Networks, 37 (2001), 171-193.

[4] D.S. Hochbaum, A. Chen, Performance analysis and best implementations of old and new algorithms for the open-pit mining problem, Operations Research, 48 (2000), 894-914.

[5] H. Lerchs, I.F. Grossmann, Optimum design of open-pit mines, Transactions, Canadian Institute of Mining and Metallurgy, LXVIII (1965), $17-24$. 
[6] J.-C. Picard, Maximal closure of a graph and applications to combinatorial problems, Management Science, 22 (1976), 1268-1272.

[7] J.-C. Picard, M. Queyranne, Selected applications of minimum cuts in networks, INFOR Journal, 20 (1982), 394-422.

[8] J.-C. Picard, B.T. Smith, Parametric maximum flows and the calculation of optimal intermediate contours in open pit mine design, INFOR Journal, 42 (2004), 143-153.

[9] J.M.W. Rhys, A selection problem of shared fixed costs and networks flows, Management Science, 17 (1970), 200-207.

[10] B. Tachefine, F. Soumis, Maximal closure on a graph with resource constraints, Computers and Operations Research, 24 (1997), 981-990. 
